\title{
Aspergillus Endocarditis in a Diabetic Patient with Brucellosis: A Case Report
}

Arif Maqsood Ali", Azhar Mahmood Kayani, Agha Babar Hussain and Shazia Arif

Department of Cardiology, Rawalpindi Institute of Cardiology, Pakistan

*Corresponding author: Ali AM, Department of Cardiology, Rawalpindi Institute of Cardiology, Pakistan, Tel: 923247312713; E-mail: drarifmaqsoodali@hotmail.com

Received: May 15, 2018; Accepted: June 07, 2018; Published: June 09, 2018

Copyright: () 2018 Ali AM, et al. This is an open-access article distributed under the terms of the creative commons attribution license, which permits unrestricted use, distribution, and reproduction in any medium, provided the original author and source are credited.

Abstract
Endocarditis is associated with significant morbidity and mortality world over especially in the developing
countries. The disease has varied presentations. Common etiological agents include Streptococcus viridians,
Coagulase negative Staphylococcus, Staphylococcus aureus and Enterococus in the culture positive endocarditis.
Rarely fungi may also lead to vegetations which are often large in size and require surgery beside medical
treatment. We present a case case report of endocarditis due to Aspergillus species with Brucellosis presented is in
a lady treated with valvular replacement and antifungal therapy with voriconazole.

Keywords: Aspergillus; Endocarditis; Vegetation

\section{Introduction}

Endocarditis is associated with significant morbidity \& mortality world over especially in the developing countries. It is not a single disease, but rather may have different presentations depending on the organs involved, the underlying cardiac disease (if any), the causative microorganism, the presence or absence of complications and the patient's characteristics [1]. Fungal valve endocarditis is rare especially when blood cultures are negative [2]. There are few reported cases of endocarditis due to Aspergillus species. The case report presented is in a young non-intravenous drug user treated with valvular replacement and antifungal therapy with voriconazole.

\section{Case Presentation}

A 38-years-old female from rural background of Punjab presented with history of cough and fever for 1 month. She got various general practitioner treatments but did not improve. She was admitted Rawalpindi Institute of Cardiology in Rawalpindi, Pakistan with above mentioned complaints. She was febrile with temperature of $99^{\circ} \mathrm{F}$. Her blood pressure was $110 / 70 \mathrm{mmHg}$. The chest was clear with bilateral vesicular breathing. She was anemic. She had systolic murmur at aortic area and diastolic murmur at mitral area. Her $\mathrm{Hb}$ was $8.7 \mathrm{~g} / \mathrm{dl}$, total leucocyte count $12.8 \times 109 / \mathrm{ul}$, ESR was $130 \mathrm{~mm} / \mathrm{hr}$. CRP was $172 \mathrm{~g} / \mathrm{dl}$. RA factor was negative. Her blood glucose was $251 \mathrm{mg} / \mathrm{dl}$. She had normal cardiac, liver and coagulation profile. She had urea $82 \mathrm{mg} / \mathrm{dl}$ and creatinine $1.3 \mathrm{mg} / \mathrm{dl}$. Transesophageal Echocardiography showed severe mitral stenosis, moderate mitral regurgitation and severe aortic stenosis. The left atrium was dilated and normal sized left ventricle. Two freely mobile vegetations $17 \mathrm{~mm} \times 12 \mathrm{~mm}$ attached to the posterior mitral leaflet on the left atrial side. Another vegetation 12 $\mathrm{mm}$ in size was seen at right coronary cusp area (Figure 1). Three sets of blood cultures were taken and empirical therapy with Vancomycin, Ceftriaoxine and Levofloxacin started. Blood cultures remained negative. However, she remained febrile with no change in size of vegetation. Her serological test for Coxiella burnetti was negative but positive for Brucella abortus. Vancomycin, ceftriaxone and
Levofloxacin were stopped and antifungal therapy with intravenous Voriconazole was started to cater for the possibility of fungal endocarditis, Doxycycline and Rifampicin for possible brucellosis. She became afebrile after a few days and size of vegetations also decreased. Her ESR and CRP also followed a decreasing trend. Because of the large sized vegetations with the risk of embolization it was decided to operate the patient rather to treat her on medical management alone. Double valve replacement of both mitral and aortic valve was performed. The vegetations were subcultured and observed. A greenish colored growth of Aspergillus species was isolated. Microscopy showed hyphae, conidiophores and conidia. (Figures 2 and 3). Oral voriconazole $200 \mathrm{mg}$ twice daily was continued for 8 weeks with follow up for any complication or relapse of vegetation. Patient remained afebrile and is monitored monthly.
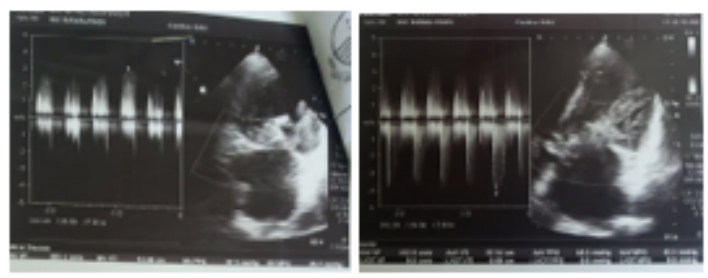

Figure 1: Transesophageal echocardiography showed dilated left atrium, normal sized left ventricle. Two vegetations $17 \times 12 \mathrm{~mm}$ attached to the posterior mitral leaflet on the left atrial side, freely mobile seen. Another vegetation $12 \mathrm{~mm}$ in size seen at RCC.

\section{Discussion}

Endocarditis is of interest to the medical specialists, cardiologists and cardiac surgeons due to its varied presentations. The diagnosis can be straight forward in a few patients with typical valvular heart disease and fever. However, often the typical text book presentation and typical signs are missing at presentation and sometimes it is complicated by its complications. Durack and his colleagues from Dukes University in 
1994 proposed criteria for its diagnosis based on the presence or absence of major and minor criteria [3].

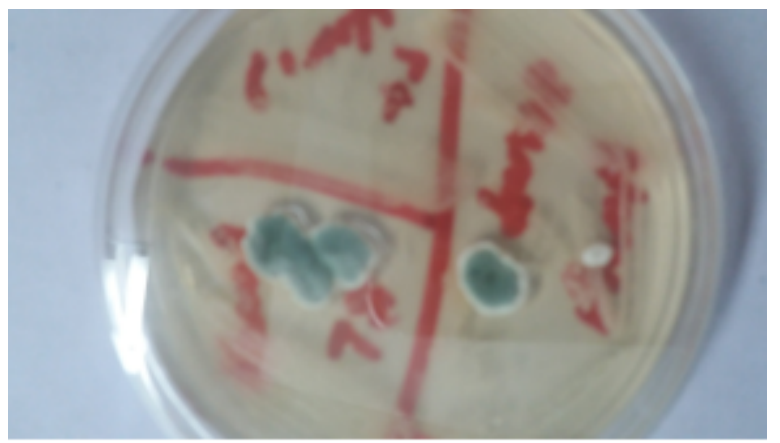

Figure 2: Growth of vegetation on sabouraud dextrose agar.

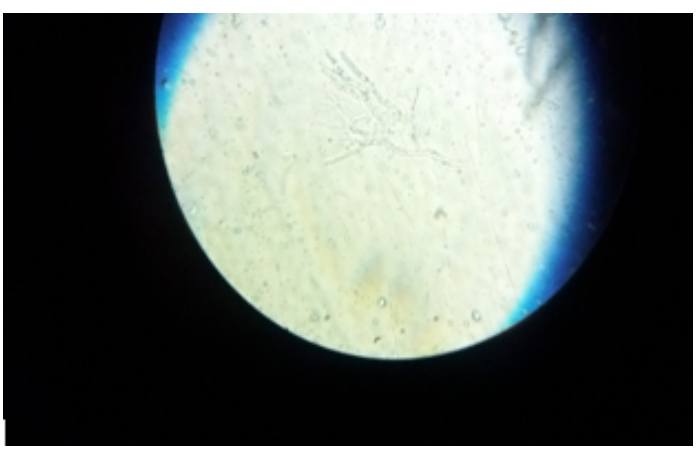

Figure 3: Microscopy showing hyphae, conidiophores and conidia.

It has been validated in a number of trials and studies and modified to include serological evidence and Staphylococcal bacteremia. The presentation can be acute in onset or chronic. Based on the vegetations it can involve the right or the left side of heart. It can be native valve or prosthetic valve endocarditis. The common causatives are Streptococcus viridians, Enrerococci, Staphylococci and less commonly fungi. Often the vegetations are large and rapid in onset especially in Staphylococcal endocarditis. It could be chronic in cases of fungal endocarditis. The most common fungi isolated are Candida species, Aspergillus and another endemic mycosis [4]. Often the cultures remain negative and diagnosis is evidenced by serological tests e.g. Coxiella burnetii, brucellosis or HACEK like organisms. Due to large sized vegetations in Staphylococcal aureus and fungal endocarditis early treatment and surgery is often required due to avoid septic embolization [3].

The empirical therapy for fungal endocarditis includes Amphotericin, Caspofungin or different azoles. Aspergillus endocarditis or endemic fungi are less common than Candida endocarditis. Fungal endocarditis can develop in immunocompromised patients, intravenous drug users and those with different cardiac devices or central venous line catheters. The mortality rate with fungal endocarditis remains high. Two phase treatments have evolved. Initial induction phase consists of antifungal therapy combined with valve surgery. Antifungal therapy is often given for greater than 6 weeks followed by suppression therapy due to high relapse rate. Patients that are unfit for surgery can be treated with long term oral antifungals with follow up [4].

Postoperatively, the vegetation yielded growth of Aspergillus species and voriconazole $200 \mathrm{mg}$ twice daily was started for a period of thirteen weeks with an excellent clinical response.

Aspergillus species can cause severe widespread invasive infections beside infection in sinuses and lungs. It can invade also heart, and central nervous system. Aspergillus most commonly infects immunocompromised hosts in the respiratory tract. Aspergillus endocarditis is relatively uncommon. Our patient had history of rural background and exposure to brucella in the past with grade 2 renal disease. Aspergillus species account for approximately 20 to $30 \%$ of all fungal endocarditis cases [5,6]. Ratio of Aspergillus to Candida (1:2) has also remained constant over the last three decades [5].

\section{Conclusion}

Successful treatment of endocarditis requires the combination of antifungal therapy and surgical debridement. The recommended antifungal therapy for most invasive Aspergillus infections, including Aspergillus endocarditis, is voriconazole [7]. The superiority of voriconazole to amphotericin B deoxycholate was demonstrated in a large, randomized controlled trial of invasive Aspergillus infections, the majority of which involved the lungs and sinuses (92\%). Voriconazole was associated with improved survival, and less nephrotoxicity, electrolyte abnormalities, and infusion-related events than with Amphotericin [8]. An Intravenous liposomal amphotericin formulation is an alternative treatment option, with equal efficacy and less nephrotoxicity than amphotericin B in the treatment of invasive Aspergillus infections [9]. Finally, surgical debridement is imperative for the survival of almost all cases of Aspergillus endocarditis.

\section{References}

1. Habib G, Lancellotti P, Antunes MJ, Bongiorni MG, Casalta JP, et al. (2015) ESC guidelines for the management of infective endocarditis. Eur Heart J 36: 3075-3128.

2. Vassiloyanakopoulos A, Falagas ME, Allamani M, Michalopoulos A (2006) Aspergillus fumigatus tricuspid native valve endocarditis in a nonintravenous drug user. J Med Microbiol 55: 635-638.

3. Councils ES (2015) ESC guidelines for the management of infective endocarditis pp: 1-3117.

4. Baddour LM, Wilson WR, Bayer AS, Fowler VG, Tleyjeh IM, et al. (2015) Infective endocarditis in adults: Diagnosis, antimicrobial therapy, and management of complications. Circulation 132: 1435-1486.

5. Ellis ME, Al-Abdely H, Sandridge A, Greer W, Ventura W (2001) Fungal endocarditis: Evidence in the world literature. Clin Infect Dis 32: 50-62.

6. Pierrotti LC, Baddour LM (2002) Fungal endocarditis. Chest 122: 302-310.

7. Walsh TJ, Anaissie EJ, Denning DW, Herbrecht R, Kontoyiannis DP, et al. (2008) Treatment of aspergillosis: Clinical practice guidelines of the infectious diseases society of america. Clin Infect Dis 46: 327-360.

8. Herbrecht R, Denning DW, Patterson TF, Bennett JE, Greene RE, et al. (2002) Voriconazole versus amphotericin B for primary therapy of invasive aspergillosis. $\mathrm{N}$ Engl J Med 347: 408-415.

9. Bowden R, Chandrasekar P, White MH, Li X, Pietrelli L, et al. (2002) A double-blind, randomized, controlled trial of amphotericin $\mathrm{B}$ colloidal dispersion versus amphotericin $B$ for treatment of invasive aspergillosis in immunocompromised patients. Clin Infect Dis 35: 359-366. 